\title{
Update to CDC's Treatment Guidelines for Gonococcal Infection, 2020
}

\author{
Sancta St. Cyr, MD ${ }^{1}$; Lindley Barbee, MD ${ }^{1,2}$; Kimberly A. Workowski, MD ${ }^{1,3}$; Laura H. Bachmann, $\mathrm{MD}^{1}$; Cau Pham, PhD ${ }^{1}$; Karen Schlanger, PhD ${ }^{1}$; \\ Elizabeth Torrone, $\mathrm{PhD}^{1}$; Hillard Weinstock, $\mathrm{MD}^{1}$; Ellen N. Kersh, $\mathrm{PhD}^{1}$; Phoebe Thorpe, $\mathrm{MD}^{1}$
}

Sexually transmitted infections (STIs) caused by the bacteria Neisseria gonorrhoeae (gonococcal infections) have increased 63\% since 2014 and are a cause of sequelae including pelvic inflammatory disease, ectopic pregnancy, and infertility and can facilitate transmission of human immunodeficiency virus (HIV) $(1,2)$. Effective treatment can prevent complications and transmission, but $N$. gonorrhoeae's ability to acquire antimicrobial resistance influences treatment recommendations and complicates control (3). In 2010, CDC recommended a single $250 \mathrm{mg}$ intramuscular (IM) dose of ceftriaxone and a single $1 \mathrm{~g}$ oral dose of azithromycin for treatment of uncomplicated gonococcal infections of the cervix, urethra, and rectum as a strategy for preventing ceftriaxone resistance and treating possible coinfection with Chlamydia trachomatis (4). Increasing concern for antimicrobial stewardship and the potential impact of dual therapy on commensal organisms and concurrent pathogens (3), in conjunction with the continued low incidence of ceftriaxone resistance and the increased incidence of azithromycin resistance, has led to reevaluation of this recommendation. This report, which updates previous guidelines (5), recommends a single $500 \mathrm{mg}$ IM dose of ceftriaxone for treatment of uncomplicated urogenital, anorectal, and pharyngeal gonorrhea. If chlamydial infection has not been excluded, concurrent treatment with doxycycline (100 mg orally twice a day for 7 days) is recommended. Continuing to monitor for emergence of ceftriaxone resistance through surveillance and health care providers' reporting of treatment failures is essential to ensuring continued efficacy of recommended regimens.

Combination therapy, using a highly effective gonococcal therapeutic agent with cotreatment for chlamydia, has been recommended since 1985. In 2007, based on data from CDC's Gonococcal Isolate Surveillance Project* (GISP) indicating

\footnotetext{
*2018 GISP sites: Albuquerque, New Mexico (1987-2018); Anchorage, Alaska (1987-2003, 2018); Atlanta, Georgia (1987-2018); Birmingham, Alabama (1987-2018); Boston, Massachusetts (1987-1992, 2014-2018); Buffalo, New York (2014-2018); Chicago, Illinois (1996-2018); Cleveland, Ohio (19912018); Columbus, Ohio (2012-2018); Dallas, Texas (1999-2018); Denver, Colorado (1987-2013, 2018); Greensboro, North Carolina (2002-2018); Honolulu, Hawaii (1987-2018); Indianapolis, Indiana (2013-2018); Jackson, Mississippi (2018); Kansas City, Missouri (1992-2001, 2007-2018); Las Vegas, Nevada (2002-2018); Los Angeles, California (2003-2018); Miami, Florida (1998-2013, 2018); Milwaukee, Wisconsin (2018); Minneapolis, Minnesota (1992-2018); New Orleans, Louisiana (1987-2018); New York, New York (2006-2018); Orange County, California (1991-2018); Phoenix, Arizona (1987-2018); Philadelphia, Pennsylvania (1987-2018); Pontiac, Michigan (2012-2018); Portland, Oregon (1987-2018); San Diego, California (19872018); San Francisco, California (1987-2018); Seattle, Washington (1987-2018); Tripler Army Medical Center, Hawaii (2001-2018); and Washington, DC (2018).
}

widely disseminated quinolone-resistant gonococcal strains in the United States, CDC no longer recommended fluoroquinolones for treatment, leaving cephalosporins as the only remaining recommended antimicrobial class (G). Availability of sensitive $C$. trachomatis nucleic acid amplification tests were widespread by 2010 , but CDC recommended gonococcal dual therapy with a cephalosporin (ceftriaxone $250 \mathrm{mg}$ IM or cefixime $400 \mathrm{mg}$ orally) and either azithromycin or doxycycline (4) to reflect concerns regarding emerging gonococcal resistance. By 2011, the minimum inhibitory concentrations (MICs) of cefixime necessary to inhibit $N$. gonorrhoeae growth in vitro were increasing. In 2012, cefixime was no longer a recommended gonococcal regimen ( 7$)$, with ceftriaxone and azithromycin combination therapy the only recommended regimen for uncomplicated gonorrhea (5). Since publication of the 2015 Sexually Transmitted Diseases (STD) Treatment Guidelines, concerns regarding antimicrobial stewardship have increased, especially the impact of antimicrobial use on the microbiome and data indicating azithromycin resistance (elevated MICs) for gonorrhea and other organisms $(1,3)$. Pharmacokinetic and pharmacodynamic modeling has also affected the understanding of optimal antimicrobial dosing for $N$. gonorrhoeae treatment. This update provides the rationale for the change in gonorrhea treatment recommendations to a higher dose $(500 \mathrm{mg})$ of ceftriaxone and removal of azithromycin from the recommended regimen.

During 2018, CDC staff members and subject matter experts identified essential questions regarding gonorrhea treatment to update the 2015 STD Treatment Guidelines (5). A literature search of PubMed, Embase, and Medline databases conducted for January 2013-May 2019 using the parameters (gonorrhea[MeSH]) OR (gonococcal[all fields] OR gonorrhea[all fields] OR "Neisseria gonorrhoeae"[all fields]) AND (treatment[MeSH] OR antibiotic[MeSH] OR therapy) generated $>2,200$ abstracts. Titles and abstracts were assessed, and 248 clinically relevant articles were reviewed. Abstracts from STD conferences held during 2015-2018 and on the National Institutes of Health clinical trials website (https:// clinicaltrials.gov) were also reviewed.

GISP susceptibility data from January 2013 to May 2019 were reviewed. GISP monitors gonorrhea antimicrobial susceptibility patterns in the United States through monthly testing of urethral isolates from 25 symptomatic men in each of 25-30 STD specialty care clinics (1). Regional laboratories 
conduct antimicrobial susceptibility testing by agar dilution to determine MICs for selected antimicrobials. Although the Clinical and Laboratory Standards Institute (CLSI) has not established $N$. gonorrhoeae resistance breakpoints for ceftriaxone, cefixime, or azithromycin, CLSI categorizes isolates with MICs of $\leq 0.25 \mu \mathrm{g} / \mathrm{mL}$ as susceptible for ceftriaxone and cefixime, and those with MICs of $\leq 1.00 \mu \mathrm{g} / \mathrm{mL}$ as susceptible for azithromycin $(8,9)$. To identify isolates with elevated MICs, GISP uses the following "alert values" to identify potential emerging resistance: $\mathrm{MIC} \geq 0.125 \mu \mathrm{g} / \mathrm{mL}$ for ceftriaxone, $\geq 0.25 \mu \mathrm{g} / \mathrm{mL}$ for cefixime, and $\geq 2 \mu \mathrm{g} / \mathrm{mL}$ for azithromycin (1).

In 2019, during an in-person meeting of governmental and nongovernmental participants, CDC staff members and subject matter experts reviewed data and presented their individual expert opinions. Each essential question was discussed, and applicable published articles were reviewed for their strengths, weaknesses, and relevance. Individual participants evaluated the quality of evidence, provided their input, and discussed findings in the context of the modified rating system used by the U.S. Preventive Services Task Force. ${ }^{\dagger}$ CDC staff members independently reviewed tables of evidence, ${ }^{\S}$ individual comments from the participants and professional organizations, and existing guidelines from other organizations to determine if revisions to the 2015 CDC STD Treatment Guidelines were warranted.

\section{Evidence and Rationale}

Antimicrobial stewardship. The 2019 report on antimicrobial resistance threats in the United States (3) highlights that antimicrobial stewardship, i.e., the development, promotion, and implementation of activities to ensure the appropriate use of antimicrobials, remains a major public health concern. Data continue to document the impact of antimicrobials on the microbiome and on pathogenic organisms. A recent investigation comparing children who received twice-yearly azithromycin with children who received placebo found that the gut's resistome, a reservoir of antimicrobial resistance genes in the body, had increased determinants of macrolide and nonmacrolide resistance, including beta-lactam antibiotics, among children receiving azithromycin (10). A higher proportion of macrolide resistance in nasopharyngeal Streptococcus pneumoniae was demonstrated in communities receiving mass administration of oral azithromycin (11). Azithromycin resistance has been demonstrated in another STI, Mycoplasma genitalium, and sexually transmissible enteric pathogens (e.g., Shigella and Campylobacter) (12-14). In addition, evidence supports increasing concern for the efficacy of azithromycin to treat chlamydial infections, especially rectal infections $(15,16)$.

\footnotetext{
${ }^{\dagger}$ https://www.uspreventiveservicestaskforce.org/uspstf/grade-definitions.

${ }^{\S}$ https://www.cdc.gov/std/treatment-guidelines/evidence.htm.
}

GISP data show that the ceftriaxone MIC50 and MIC90 (MIC required to inhibit growth of $50 \%$ and $90 \%$ of organisms, respectively) were only one doubling dilution higher during 2014-2018, compared with the respective ceftriaxone MIC50 and MIC90 during 1992-1995 (1). Although dual drug therapy with different mechanisms of action (ceftriaxone and azithromycin) might have mitigated emergence of reduced susceptibility to ceftriaxone in $N$. gonorrhoeae, concerns regarding potential harm to the microbiome and the effect on other pathogens diminishes the benefits of maintaining dual therapy as the recommended treatment regimen.

Pharmacokinetic and pharmacodynamic considerations. Ceftriaxone is a bactericidal third-generation cephalosporin with widely variable pharmacokinetics (17). Efficacy is best predicted by time that the serum free (i.e., unbound) drug concentration remains higher than the organism's MIC $\left(f \Gamma_{>M I C}\right)$. Although no human data exist confirming the length of time above the MIC required to eradicate gonorrhea at different anatomic sites, using Monte Carlo modeling, ceftriaxone has been estimated to require concentrations higher than the strain MIC for approximately 20-24 hours for effective urogenital gonorrhea treatment (18). A $250 \mathrm{mg}$ ceftriaxone dose does not reliably achieve levels higher than an $\mathrm{MIC} \geq 0.125 \mu \mathrm{g} / \mathrm{mL}$ for an extended duration (18). A murine model was used to estimate pharmacokinetic and pharmacodynamic parameters needed for cure at urogenital sites for both susceptible and resistant strains of $N$. gonorrhoeae (19). Investigators evaluated the efficacy of various ceftriaxone doses $(0.06-30 \mathrm{mg} / \mathrm{kg}$ body weight). The lowest ceftriaxone dose that was $100 \%$ effective at eradicating the susceptible organism ( $\mathrm{MIC}=0.008 \mu \mathrm{g} / \mathrm{mL}) 48$ hours after treatment was $5 \mathrm{mg} / \mathrm{kg}$ body weight, which corresponded to an $f \Gamma_{>\text {MIC }}$ of 23.6 hours, consistent with the Monte Carlo simulation $(18,19)$. Translating into human doses, a 500-mg dose corresponds to $5 \mathrm{mg} / \mathrm{kg}$ body weight $(80-100 \mathrm{~kg}$ ) human, whereas $250 \mathrm{mg}$ only corresponds to $3 \mathrm{mg} / \mathrm{kg}$ body weight for an $80 \mathrm{~kg}$ person.

The pharynx tends to be screened less often (1) than other anatomic sites, and globally, most reported ceftriaxone-based regimen treatment failures have involved treatment of pharyngeal gonorrhea (20). Ceftriaxone concentrations tend to be more variable in the pharynx, and treatment of $N$. gonorrhoeae likely requires longer times above the strain's $\operatorname{MIC}(21,22)$. Continued uncertainty regarding ceftriaxone pharmacokinetics and pharmacodynamics in treating pharyngeal gonorrhea and the higher likelihood of treatment failures at this site strengthen the recommendation for an increase in the ceftriaxone dosage to $500 \mathrm{mg}$.

Changes in azithromycin susceptibility. Azithromycin resistance in $N$. gonorrhoeae is an increasing concern. Genomic epidemiology data confirm that azithromycin resistance can result from multiple mechanisms (23). Nationally, the 
FIGURE. Percentage of Neisseria gonorrhoeae isolates with elevated minimum inhibitory concentrations (MICs)* to ceftriaxone, cefixime, and azithromycin — Gonococcal Isolate Surveillance Project, United States, 2009-2018

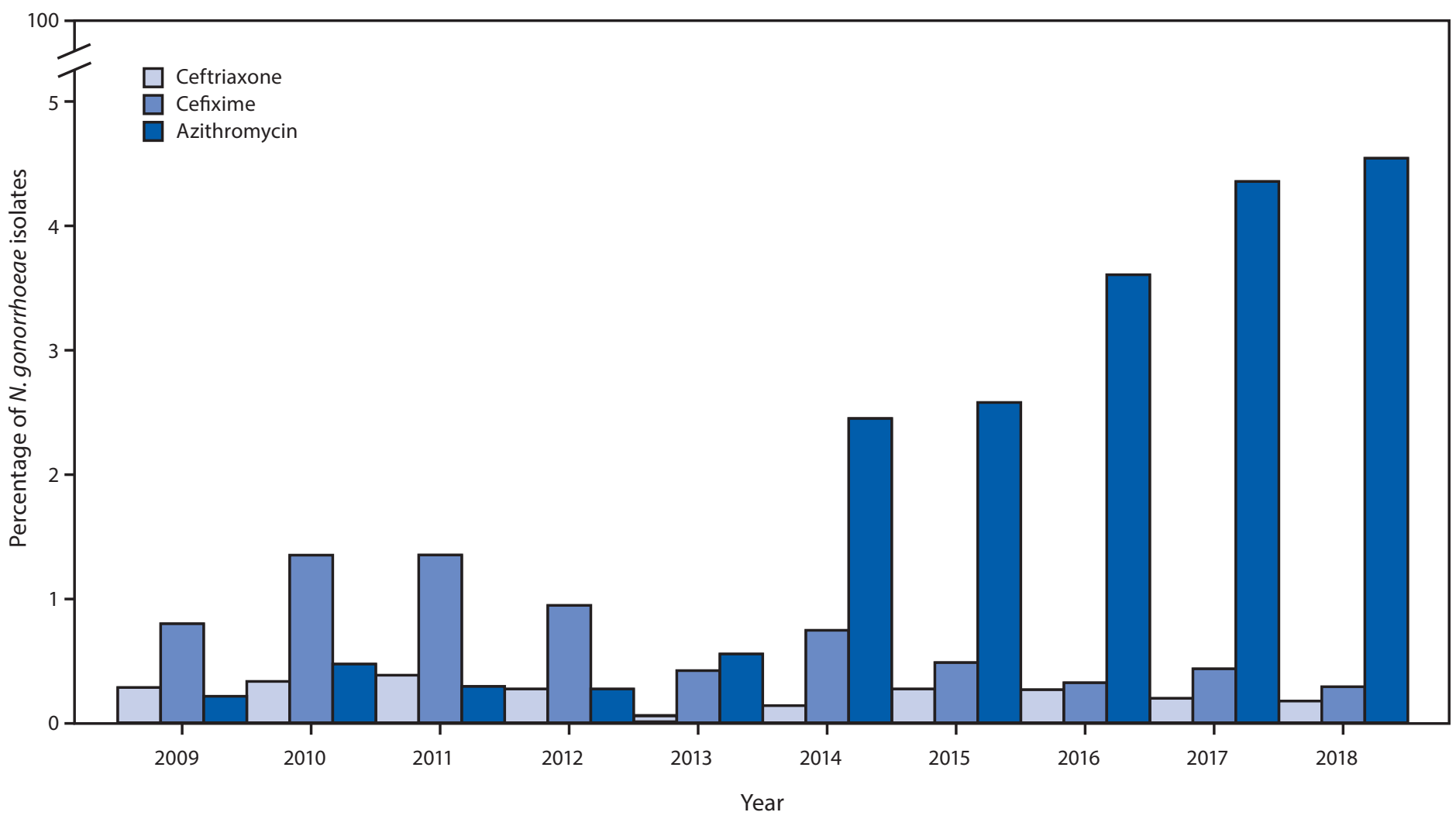

Source: CDC. Sexually Transmitted Disease Surveillance 2018. https://www.cdc.gov/std/stats18/default.htm. * Elevated MIC = ceftriaxone $\geq 0.125 \mu \mathrm{g} / \mathrm{mL}$; cefixime $\geq 0.25 \mu \mathrm{g} / \mathrm{mL}$; azithromycin $\geq 2.0 \mu \mathrm{g} / \mathrm{mL}$.

percentage of $N$. gonorrhoeae isolates with reduced susceptibility (MIC $\geq 2.0 \mu \mathrm{g} / \mathrm{mL}$ ) increased more than sevenfold over 5 years (from $0.6 \%$ in 2013 to $4.6 \%$ in 2018) (Figure) (1). During 2018, among men who have sex with men, the proportion of GISP isolates with an azithromycin alert value was $8.6 \%$, compared with $2.9 \%$ among men who have sex with women only (1). Studies have associated development of reduced azithromycin susceptibility with azithromycin exposure among patients with $N$. gonorrhoeae infection $(24,25)$.

\section{Recommendations}

For treatment of uncomplicated urogenital, rectal, or pharyngeal gonorrhea, CDC recommends a single $500 \mathrm{mg}$ IM dose of ceftriaxone (Box). For persons weighing $\geq 150 \mathrm{~kg}$ (300 lbs), a single $1 \mathrm{~g}$ IM dose of ceftriaxone should be administered. If chlamydial infection has not been excluded, doxycycline 100 mg orally twice a day for 7 days is recommended. When ceftriaxone cannot be used for treating urogenital or rectal gonorrhea because of cephalosporin allergy, a single $240 \mathrm{mg}$ IM dose of gentamicin plus a single $2 \mathrm{~g}$ oral dose of azithromycin is an option. Gastrointestinal symptoms, primarily vomiting within 1 hour of dosing, have been reported among 3\%-4\% of treated persons (26). If administration of IM ceftriaxone is not available, a single $800 \mathrm{mg}$ oral dose of cefixime is an alternative regimen. However, cefixime does not provide as high, or as sustained, bactericidal blood levels as does ceftriaxone and demonstrates limited treatment efficacy for pharyngeal gonorrhea $(27,28)$.

In cases where gonococcal expedited partner therapy (provision of prescriptions or medications for the patient to take to a sex partner without the health care provider first examining the partner) is permissible by state law and the partner is unable or unlikely to seek timely treatment, the partner may be treated with a single $800 \mathrm{mg}$ oral dose of cefixime, provided that concurrent chlamydial infection in the patient has been excluded. Otherwise, the partner may be treated with a single oral $800 \mathrm{mg}$ cefixime dose plus oral doxycycline $100 \mathrm{mg}$ twice daily for 7 days.

In cases of suspected cephalosporin treatment failure, clinicians should obtain relevant clinical specimens for culture and antimicrobial susceptibility testing, consult an infectious disease specialist or STD clinical expert (https://www.stdccn.org/) 
BOX. CDC recommended regimens for uncomplicated gonococcal infections, 2020

Regimen for uncomplicated gonococcal infections of the cervix, urethra, or rectum:

Ceftriaxone $500 \mathrm{mg}$ IM as a single dose for persons weighing $<150 \mathrm{~kg}$ (300 lb).

- For persons weighing $\geq 150 \mathrm{~kg}$ (300 lb), $1 \mathrm{~g}$ of IM ceftriaxone should be administered.

- If chlamydial infection has not been excluded, providers should treat for chlamydia with doxycycline $100 \mathrm{mg}$ orally twice daily for 7 days. During pregnancy, azithromycin $1 \mathrm{~g}$ as a single dose is recommended to treat chlamydia.

\section{Alternative regimens for uncomplicated gonococcal infections of the cervix, urethra, or rectum if ceftriaxone is not available:}

Gentamicin $240 \mathrm{mg}$ IM as a single dose plus azithromycin $2 \mathrm{~g}$ orally as a single dose OR

Cefixime $800 \mathrm{mg}$ orally as a single dose. If treating with cefixime, and chlamydial infection has not been excluded, providers should treat for chlamydia with doxycycline $100 \mathrm{mg}$ orally twice daily for 7 days. During pregnancy, azithromycin $1 \mathrm{~g}$ as a single dose is recommended to treat chlamydia.

\section{Recommended regimen for uncomplicated gonococcal infections of the pharynx:}

Ceftriaxone $500 \mathrm{mg}$ IM as a single dose for persons weighing $<150 \mathrm{~kg}(300 \mathrm{lb})$.

- For persons weighing $\geq 150 \mathrm{~kg}$ (300 lb), $1 \mathrm{~g}$ of IM ceftriaxone should be administered.

- If chlamydia coinfection is identified when pharyngeal gonorrhea testing is performed, providers should treat for chlamydia with doxycycline $100 \mathrm{mg}$ orally twice a day for 7 days. During pregnancy, azithromycin $1 \mathrm{~g}$ as a single dose is recommended to treat chlamydia.

- No reliable alternative treatments are available for pharyngeal gonorrhea. For persons with a history of a beta-lactam allergy, a thorough assessment of the reaction is recommended.*

- For persons with an anaphylactic or other severe reaction (e.g., Stevens Johnson syndrome) to ceftriaxone, consult an infectious disease specialist for an alternative treatment recommendation.

Abbreviation: IM = intramuscular.

${ }^{*}$ CDC. Sexually transmitted diseases treatment guidelines. MMWR Recomm Rep 2015;64(No. RR-3). https://www.cdc.gov/mmwr/preview/ mmwrhtml/rr6403al.htm.

\section{Summary}

What is already known about this topic?

Neisseria gonorrhoeae is an important cause of sexually transmitted infections that can have severe reproductive health consequences. N. gonorrhoeae can rapidly develop antibiotic resistance.

What is added by this report?

Based on review of recent evidence, $C D C$ recommends a single $500 \mathrm{mg}$ intramuscular dose of ceftriaxone for uncomplicated gonorrhea. Treatment for coinfection with Chlamydia trachomatis with oral doxycycline ( $100 \mathrm{mg}$ twice daily for 7 days) should be administered when chlamydial infection has not been excluded.

What are the implications for public health practice?

Continuing to monitor for emergence of ceftriaxone resistance will be essential to ensuring continued efficacy of recommended regimens.

for guidance in clinical management, and report the case to CDC through state and local public health authorities within 24 hours. Health departments should prioritize notification and culture evaluation for the patient's sex partner(s) from the preceding 60 days for those with suspected cephalosporin treatment failure or persons whose gonococcal isolates demonstrate reduced susceptibility to cephalosporins.

A test-of-cure is unnecessary for persons with uncomplicated urogenital or rectal gonorrhea who are treated with any of the recommended or alternative regimens; however, for persons with pharyngeal gonorrhea, a test-of-cure is recommended, using culture or nucleic acid amplification tests $7-14$ days after initial treatment, regardless of the treatment regimen. Because reinfection within 12 months ranges from $7 \%$ to $12 \%$ among persons previously treated for gonorrhea $(29,30)$, persons who have been treated for gonorrhea should be retested 3 months after treatment regardless of whether they believe their sex partners were treated. If retesting at 3 months is not possible, clinicians should retest within 12 months after initial treatment.

\section{Discussion}

Continued support of gonorrhea prevention and control efforts remains fundamental, and preventing antibiotic resistance is crucial. The pharmacokinetics and pharmacodynamics of ceftriaxone indicate that a $500 \mathrm{mg}$ dose in an average-weight U.S. adult achieves sufficiently high serum levels for an adequate duration to eradicate infection, even with wide pharmacokinetic variability. The high frequency of pharyngeal gonorrhea with substantial underscreening and the increased understanding of wide individual pharmacokinetic and pharmacodynamic variability has contributed to the recommendation for the increased ceftriaxone dose. These recommendations also include a test-of-cure for persons with 
pharyngeal gonorrhea to ensure eradication or detection of a possible treatment failure.

Emerging antimicrobial resistance affects gonorrhea treatment recommendations and other STIs. CDC recommends ceftriaxone monotherapy for treatment because $N$. gonorrhoeae remains highly susceptible to ceftriaxone, azithromycin resistance is increasing, and prudent use of antimicrobial agents supports limiting their use. Continuing to monitor for emergence of ceftriaxone resistance through surveillance and health care providers' reporting of treatment failures will be essential to ensuring continued efficacy of recommended regimens.

\section{Acknowledgments}

Gail A. Bolan, MD, Division of STD Prevention, National Center for HIV/AIDS, Viral Hepatitis, STD, and TB Prevention, CDC; 2018 Antibiotic Resistance Laboratory Network Regional Gonorrhea Laboratories: Maryland Department of Health and Mental Hygiene, Tennessee Department of Health, Texas Department of State Health Services, Washington State Department of Health.

\section{Gonorrhea Treatment Workgroup}

H. Hunter Handsfield, MD, Department of Medicine, University of Washington, Seattle, Washington; Edward W. Hook III, MD, Division of Infectious Diseases, University of Alabama at Birmingham, Birmingham, Alabama; Jeffrey D. Klausner, MD, Division of Infectious Diseases, University of California at Los Angeles, Los Angeles, California; Olusegun O. Soge, PhD, Department of Medicine, University of Washington, Seattle, Washington; Stephanie Taylor, MD, Department of Medicine, Louisiana State University, New Orleans, Louisiana; Magnus Unemo, PhD, School of Medical Sciences, Örebro University, Örebro, Sweden; Jonathan M. Zenilman, MD, Department of Medicine, Johns Hopkins University, Baltimore, Maryland.

Corresponding author: Laura H. Bachmann, LBachmann@cdc.gov, 404-718-5053.

${ }^{1}$ Division of STD Prevention, National Center for HIV/AIDS, Viral Hepatitis, STD, and TB Prevention, CDC; ${ }^{2}$ Department of Medicine, University of Washington, Seattle, Washington; ${ }^{3}$ Department of Medicine, Emory University, Atlanta, Georgia.

All authors have completed and submitted the International Committee of Medical Journal Editors form for disclosure of potential conflicts of interest. Lindley Barbee reports a grant from SpeeDx and from Nabriva, personal fees from Nabriva, and nonfinancial support from Hologic, outside the submitted work. No other potential conflicts of interest were disclosed.

\section{References}

1. CDC. Sexually transmitted disease surveillance 2018. Atlanta, GA: US Department of Health and Human Services, CDC; 2019. https://www. cdc.gov/std/stats18/STDSurveillance2018-full-report.pdf

2. Fleming DT, Wasserheit JN. From epidemiological synergy to public health policy and practice: the contribution of other sexually transmitted diseases to sexual transmission of HIV infection. Sex Transm Infect 1999;75:3-17. PMID:10448335 https://doi.org/10.1136/sti.75.1.3
3. CDC. Antibiotic resistance threats in the United States, 2019. Atlanta, GA: US Department of Health and Human Services, CDC; 2019. https:// www.cdc.gov/drugresistance/pdf/threats-report/2019-ar-threatsreport-508.pdf

4. Workowski KA, Berman S; CDC. Sexually transmitted diseases treatment guidelines, 2010. MMWR Recomm Rep 2010;59(No. RR-12). PMID:21160459

5. Workowski KA, Bolan GA; CDC. Sexually transmitted diseases treatment guidelines, 2015. MMWR Recomm Rep 2015;64(No. RR-3). PMID:26042815

6. CDC. Update to CDC's sexually transmitted diseases treatment guidelines, 2006: fluoroquinolones no longer recommended for treatment of gonococcal infections. MMWR Morb Mortal Wkly Rep 2007;56:332-6. PMID:17431378

7. CDC. Update to CDC's Sexually transmitted diseases treatment guidelines, 2010: oral cephalosporins no longer a recommended treatment for gonococcal infections. MMWR Morb Mortal Wkly Rep 2012;61:590-4. PMID:22874837

8. Clinical and Laboratory Standards Institute. Performance standards for antimicrobial susceptibility testing. 30th ed. Wayne, PA: Clinical and Laboratory Standards Institute; 2020. https://clsi.org/media/3481/ m100ed30_sample.pdf

9. Kersh EN, Allen V, Ransom E, et al. Rationale for a Neisseria gonorrhoeae susceptible-only interpretive breakpoint for azithromycin. Clin Infect Dis 2020;70:798-804. PMID:30963175

10. Doan T, Worden L, Hinterwirth A, et al. Macrolide and nonmacrolide resistance with mass azithromycin distribution. N Engl J Med 2020;383:1941-50. PMID:33176084 https://doi.org/10.1056/ NEJMoa2002606

11. Doan T, Arzika AM, Hinterwirth A, et al.; MORDOR Study Group. Macrolide resistance in MORDOR I-a cluster-randomized trial in Niger. N Engl J Med 2019;380:2271-3. PMID:31167060 https://doi. org/10.1056/NEJMc1901535

12. Bachmann LH, Kirkcaldy RD, Geisler WM, et al. Prevalence of Mycoplasma genitalium infection, antimicrobial resistance mutations and symptom resolution following treatment of urethritis. Clin Infect Dis 2020;ciaa293. Epub March 18, 2020.

13. Yousfi K, Gaudreau C, Pilon PA, et al. Genetic mechanisms behind the spread of reduced susceptibility to azithromycin in Shigella strains isolated from men who have sex with men in Québec, Canada. Antimicrob Agents Chemother 2019;63:e01679-18. PMID:30455248

14. Gaudreau C, Pilon PA, Sylvestre JL, Boucher F, Bekal S. Multidrugresistant Campylobacter coli in men who have sex with men, Quebec, Canada, 2015. Emerg Infect Dis 2016;22:1661-3. PMID:27533504 https://doi.org/10.3201/eid2209.151695

15. Kong FY, Tabrizi SN, Law M, et al. Azithromycin versus doxycycline for the treatment of genital chlamydia infection: a meta-analysis of randomized controlled trials. Clin Infect Dis 2014;59:193-205. PMID:24729507 https://doi.org/10.1093/cid/ciu220

16. Dombrowski JC, Wierzbicki MR, Newman L, et al. A randomized trial of azithromycin vs. doxycycline for the treatment of rectal chlamydia in men who have sex with men. Presented at the National STD Prevention Conference, Atlanta, GA: September 14-24, 2020.

17. Perry TR, Schentag JJ. Clinical use of ceftriaxone: a pharmacokineticpharmacodynamic perspective on the impact of minimum inhibitory concentration and serum protein binding. Clin Pharmacokinet 2001;40:685-94. PMID:1 1605716 https://doi. org/10.2165/00003088-200140090-00004

18. Chisholm SA, Mouton JW, Lewis DA, Nichols T, Ison CA, Livermore DM. Cephalosporin MIC creep among gonococci: time for a pharmacodynamic rethink? J Antimicrob Chemother 2010;65:2141-8. PMID:20693173 https://doi.org/10.1093/jac/dkq289 
19. Connolly KL, Eakin AE, Gomez C, Osborn BL, Unemo M, Jerse AE. Pharmacokinetic data are predictive of in vivo efficacy for cefixime and ceftriaxone against susceptible and resistant Neisseria gonorrhoeae strains in the gonorrhea mouse model. Antimicrob Agents Chemother 2019;63:e01644-18. PMID:30642924 https://doi.org/10.1128/ AAC.01644-18

20. Unemo M, Golparian D, Eyre DW. Antimicrobial resistance in Neisseria gonorrhoeae and treatment of gonorrhea. Methods Mol Biol 2019;1997:37-58. PMID:31119616 https://doi. org/10.1007/978-1-4939-9496-0_3

21. Blumer JL, Reed MD, Kaplan EL, Drusano GL. Explaining the poor bacteriologic eradication rate of single-dose ceftriaxone in group a streptococcal tonsillopharyngitis: a reverse engineering solution using pharmacodynamic modeling. Pediatrics 2005;116:927-32. PMID:16199703 https://doi.org/10.1542/peds.2004-2294

22. Moran JS, Levine WC. Drugs of choice for the treatment of uncomplicated gonococcal infections. Clin Infect Dis 1995;20(Suppl 1):S47-65. PMID:7795109 https://doi.org/10.1093/ clinids/20.Supplement_1.S47

23. Grad YH, Harris SR, Kirkcaldy RD, et al. Genomic epidemiology of gonococcal resistance to extended-spectrum cephalosporins, macrolides, and fluoroquinolones in the United States, 2000-2013. J Infect Dis 2016;214:1579-87. PMID:27638945 https://doi.org/10.1093/infdis/jiw420

24. Wind CM, de Vries E, Schim van der Loeff MF, et al. Decreased azithromycin susceptibility of Neisseria gonorrhoeae isolates in patients recently treated with azithromycin. Clin Infect Dis 2017;65:37-45. PMID:28510723 https://doi.org/10.1093/cid/cix249
25. Gernert KM, Seby S, Schmerer MW, et al.; Antimicrobial-Resistant Neisseria gonorrhoeae Working Rroup. Azithromycin susceptibility of Neisseria gonorrhoeae in the USA in 2017: a genomic analysis of surveillance data. Lancet Microbe 2020;1:e154-64. PMID:33005903 https://doi.org/10.1016/S2666-5247(20)30059-8

26. Kirkcaldy RD, Weinstock HS, Moore PC, et al. The efficacy and safety of gentamicin plus azithromycin and gemifloxacin plus azithromycin as treatment of uncomplicated gonorrhea. Clin Infect Dis 2014;59:1083-91. PMID:25031289 https://doi.org/10.1093/cid/ciu521

27. Moran JS. Treating uncomplicated Neisseria gonorrhoeae infections: is the anatomic site of infection important? Sex Transm Dis 1995;22:39-47. PMID:7709324 https://doi.org/10.1097/00007435-199501000-00007

28. Gratrix J, Bergman J, Egan C, Drews SJ, Read R, Singh AE. Retrospective review of pharyngeal gonorrhea treatment failures in Alberta, Canada. Sex Transm Dis 2013;40:877-9. PMID:24113412 https://doi. org/10.1097/OLQ.0000000000000033

29. Fung M, Scott KC, Kent CK, Klausner JD. Chlamydial and gonococcal reinfection among men: a systematic review of data to evaluate the need for retesting. Sex Transm Infect 2007;83:304-9. PMID:17166889 https://doi.org/10.1136/sti.2006.024059

30. Hosenfeld CB, Workowski KA, Berman S, et al. Repeat infection with Chlamydia and gonorrhea among females: a systematic review of the literature. Sex Transm Dis 2009;36:478-89. PMID:19617871 https:// doi.org/10.1097/OLQ.0b013e3181a2a933 